\title{
VALIDAÇÃO DE UM SERIOUS GAME PARA ATENDIMENTO AO BEBÊ: ESTUDO METODOLÓGICO
}

\section{VALIDATION OF A SERIOUS GAME FOR BABY CARE: A METHODOLOGICAL STUDY}

\section{Bruna Caroline Rodrigues ${ }^{1} *$ Gabrieli Patrício Rissi $^{2} *$ Muriel Fernanda de Lima $^{3} *$ Diego $^{*}$ Raone Ferreira $^{4} *$ Roberta Tognollo Borotta Uema $^{5} *$ Ieda Harumi Higarashi $^{6}$}

\section{RESUMO}

Objetivo: validar a usabilidade e aplicabilidade de um serious game sobre assistência ao bebê menor de um ano em parada cardiorrespiratória, na perspectiva de profissionais de enfermagem de um serviço de pronto atendimento. Método: estudo metodológico que utilizou Design Participativo e o instrumento EGameFlow. Os sujeitos foram nove profissionais de enfermagem de uma Unidade de Pronto Atendimento. Os dados foram coletados por meio de grupo focal e submetidos à análise categorial temática. Resultados: todas as dimensões do instrumento EGameFlow mostraram-se adequadas. A discussão em grupo permitiu a configuração de duas categorias 1) Dificuldades vivenciadas pela equipe no uso do Reanimabebê; 2) Reanimabebê: inovação tecnológica e realismo como estratégias para a educação permanente. Os resultados foram consolidados com base na avaliação do game pelos profissionais. Considerações Finais: a ferramenta constitui-se adequada para aplicabilidade, além de ser inovadora, didática e próxima da realidade vivenciada.

Palavras-chave: Software; Tecnologia educacional; Reanimação cardiopulmonar; Pediatria; Emergências.

\begin{abstract} close to the experienced reality. Emergencies.

\footnotetext{
${ }^{1}$ Universidade Estadual de Maringá, Maringá, Brasil. https://orcid.org/0000-0003-0347-5127

${ }^{2}$ Universidade Estadual de Maringá, Maringá, Brasil. https://orcid.org/0000-0002-1702-4004

${ }^{3}$ Universidade Federal de Mato Grosso do Sul. Coxim, Brasil. https://orcid.org/0000-0001-9812-659X

${ }^{4}$ Universidade Estadual de Maringá. Maringá, Brasil. https://orcid.org/0000-0001-7633-2085

${ }^{5}$ Universidade Estadual de Maringá, Maringá, Brasil.https://orcid.org/ 0000-0002-8755-334X

${ }^{6}$ Universidade Estadual de Maringá, Maringá, Brasil. https://orcid.org/0000-0002-4205-6841
}

Objective: to validate the usability and applicability of a serious game about assistance to infants under one year of age in cardiac arrest, from the perspective of nursing professionals in an emergency care service. Method: methodological study that used Participatory Design and the EGameFlow instrument. The subjects were nine nursing professionals from an Emergency Care Unit. Data were collected through a focus group and submitted to thematic categorical analysis. Results: all dimensions of the EGameFlow instrument proved to be adequate. The group discussion allowed the configuration of two categories 1) Difficulties experienced by the team in the use of Reanimabebê; 2) Reanimabebê: technological innovation and realism as strategies for permanent education. The results were consolidated based on the game's evaluation by the professionals. Final Considerations: the tool is suitable for applicability, in addition to being innovative, didactic and

Keywords: Software; Educational technology; Cardiopulmonary resuscitation; Pediatrics; 


\section{INTRODUÇÃO}

Os serious game são utilizados para treinar e ampliar o conhecimento, discutir soluções, simular situações em diferentes contextos e conscientizar crianças e adultos na educação em saúde ${ }^{(1)}$. O jogador deve lidar com a tomada de decisão em situações críticas que são reproduzidas eletronicamente, baseadas no realismo vivenciado no cotidiano, cujo objetivo é verificar se o jogador reconhece o assunto e sabe indicar soluções $^{(2)}$.

O uso de dispositivos móveis pode servir como um complemento aos métodos tradicionais de aprendizagem, ajudando a minimizar a lacuna entre teoria e prática, permitindo o acesso à informação conforme as necessidades de cada indivíduo ${ }^{(3-4)}$.

Estudo desenvolvido na Suécia, a partir da utilização de um serious game voltado à ressuscitação cardiopulmonar, revelou que durante o treinamento, o nível de tensão mental foi de baixo a moderado, pois os estudantes afirmaram que o game promoveu a diminuição do estresse devido ao caráter fictício de jogos virtuais, ao contrário da importante apreensão experimentada frente a uma situação real de parada cardiorrespiratória $^{(5)}$.

Em revisão integrativa sobre o desenvolvimento e validação do serious game no Brasil, encontraram-se jogos que exploravam as patologias visando à promoção, prevenção, tratamento e reabilitação da saúde, voltados tanto para estudantes de graduação, profissionais da saúde e para a população em geral $^{(6)}$.

Nesse contexto, desenvolveu-se um serious game, denominado Reanimabebê, que teve como princípio a reanimação cardiopulmonar em bebês menores de um ano de vida. A escolha do tema ocorreu por uma demanda do próprio serviço em questão e surgiu por meio de um grupo focal realizado com a equipe previamente ao desenvolvimento do game.

Acredita-se que o uso do game dentro do serviço de saúde pode ajudar a diminuir a lacuna existente entre teoria e prática, além de funcionar como uma estratégia dinâmica de educação permanente. Torna-se uma estratégia inovadora e pioneira no que tange à melhora da qualidade da assistência. Diante disso, estabeleceu-se como objetivo, validar a usabilidade e aplicabilidade de um serious game sobre assistência ao bebê menor de um ano em parada cardiorrespiratória, na perspectiva de profissionais de enfermagem de um serviço de pronto atendimento.

\section{MÉTODOS}

Trata-se de um estudo de caráter metodológico, utilizando o Design Participativo (DP) ${ }^{(7)}$, cuja finalidade é propor meios para que o sujeito participe efetivamente durante o ciclo do design e desenvolvimento do projeto, possibilitando que perspectivas e necessidades do software 
sejam propostas, desenvolvidas e avaliadas por quem irá efetivamente usá-lo ${ }^{(8)}$. Para a criação do software, o estudo teve como ponto de partida as necessidades de aprendizagem da equipe de enfermagem em relação a situações emergenciais na criança, seguindo os princípios do $\mathrm{DP}^{(9)}$.

No Reanimabebê, o cenário e a narrativa do jogo acontecem em uma sala de emergência de um Pronto Atendimento à Criança situado em uma Unidade de Pronto Atendimento. O conteúdo foi desenvolvido baseado nos padrões de atendimento estabelecidos pela literatura: Pedriatric Advanced Life Support (PALS) da American Heart Association (AHA) ${ }^{(10)}$.

Os sujeitos foram profissionais de enfermagem dos diferentes turnos, selecionados intencionalmente, frente aos objetivos estabelecidos para a pesquisa, a partir dos seguintes critérios de inclusão: possuir dispositivo móvel com acesso à Internet, ter conhecimentos básicos de informática e apresentar tempo de serviço na unidade de, no mínimo, seis meses no setor infantil, considerando-se este tempo como requisito mínimo para que o profissional estivesse familiarizado com a rotina do serviço; somado ao conhecimento do pesquisador sobre a população ${ }^{(11)}$.

$\mathrm{O}$ pronto atendimento realiza atendimento às crianças de zero a 13 anos, 11 meses e 29 dias, de natureza clínica, buscando a estabilização do paciente, e inicia a investigação diagnóstica, definindo a necessidade de encaminhamento a serviços de maior complexidade. A coleta de dados ocorreu em fevereiro de 2019, por meio de um grupo focal conduzido pela pesquisadora com auxílio de um observador que realizou anotações em diário de campo, na própria instituição, em horário programado com a equipe e durou cerca de $1 \mathrm{~h}$ e $30 \mathrm{~min}$. Utilizou-se um gravador para garantir o registro fidedigno e completo das falas para posterior transcrição.

A discussão do grupo focal foi iniciada a partir da seguinte questão disparadora: Como você avaliou a experiência de utilização de uma tecnologia digital como forma/ferramenta de aprendizagem sobre parada cardiorrespiratória (PCR) no bebê menor de um ano? Empregou-se um guia de tema desenvolvido pela pesquisadora, composto por itens pautados na experiência do jogador em relação ao desempenho, manipulação, design, dinâmica, melhoria do conhecimento, dificuldades e facilidades no jogo. Na segunda parte do encontro, os sujeitos utilizaram um instrumento para avaliação do jogo, chamado EGameFlow ${ }^{(12)}$.

$\mathrm{O}$ instrumento foi traduzido, adaptado e aplicado inicialmente pelo Laboratório de Objetos de Aprendizagem da Universidade Federal de São Carlos ${ }^{(13)}$ e, posteriormente, em estudo realizado nesta mesma Universidade, no qual os autores avaliaram um serious game sobre alimentação saudável 
e exercício físico para promoção da saúde e auxílio ao enfrentamento da obesidade infantil $^{(14)}$. Baseado em uma escala de satisfação do usuário com o jogo virtual, ele auxilia a equipe que criou o jogo a perceber os pontos fortes e fracos na visão do jogador $^{(12)}$. O EGameFlow possibilita a identificação de problemas relevantes no jogo, evidenciando ser um método simplificado e efetivo para a análise detalhada de aspectos relacionados à diversão e também à aprendizagem $^{(15)}$.

O avaliador atribui a cada item, das oito dimensões existentes (Concentração, Desafios, Autonomia, Clareza dos objetivos, Feedback, Imersão, Interação Social e Melhoria do Conhecimento), uma nota condizente com sua experiência ao interagir com o jogo educacional. No Reanimabebê, a categoria "interação social" presente na versão original foi abolida, pois não contém tais características. Os escores variam de $1 \mathrm{a}$ 7, sendo 1 considerado "fraco" e o 7, "forte"; estes são então analisados para determinar a nota de cada dimensão. A nota final de cada dimensão é obtida a partir da média das notas dos itens que a compõem ${ }^{(12)}$. Consideraram-se os itens com média final igual ou abaixo de seis como itens a serem repensados para melhoria da versão final do jogo.

A nota final atribuída para cada dimensão é uma média das avaliações dos itens relacionados, considerando as notas finais atribuídas pelos avaliadores. Caso algum item fosse considerado irrelevante para o aplicativo ou não existente no game, ou quando o avaliador não se sentisse qualificado para julgar uma destas variáveis, tinha a opção de exclusão da avaliação do item, demarcada como "Não se Aplica" (NA).

Os dados oriundos dos grupos focais foram transcritos, analisados e categorizados segundo a técnica de análise de conteúdo ${ }^{(16)}$. Os dados do questionário EGameFlow foram analisados quantitativamente por estatística descritiva simples e organizados em tabela. Os participantes foram identificados com a letra $\mathrm{E}$, referente à primeira letra da palavra Enfermagem e números arábicos.

A pesquisa foi aprovada pelo Comitê de Ética em Pesquisa institucional, sob parecer $n^{\circ} 2.424 .019 / 2018$ e Certificado de Apresentação para Apreciação Ética $n^{\circ}$ 79615917.6.0000.0104. Os sujeitos foram abordados para participação mediante anuência prévia e devidamente registrada em Termo de Consentimento Livre e Esclarecido.

\section{RESULTADOS}

Participaram da pesquisa nove sujeitos, sendo três enfermeiros e seis técnicos de enfermagem. Oito eram mulheres e um, homem, com idades entre 28 e 42 anos (média de 34,5 anos); sete eram casados e dois, solteiros.

Cinco sujeitos apresentavam curso superior em enfermagem, e quatro pessoas possuíam pós-graduação. O tempo de 
formação variou de dois a 20 anos (média de 7,7 anos), com tempo de atuação na pediatria de um a seis anos (média de três anos) e tempo de trabalho na instituição entre um e três anos (média de 2,4 anos). Com relação ao turno, cinco pessoas trabalhavam no período matutino e quatro, no período vespertino.

Apenas duas pessoas referiram não ter experiência, apesar de trabalharem por um ano no serviço. Todos os profissionais afirmaram participar de capacitações. Quando questionados sobre o último curso ou capacitação de que participaram, três relataram que havia sido há cinco meses, três a três meses, um há mais de um ano e três das pessoas não se recordavam da data ou período da última instrução. Com relação à temática, dois referiram o atendimento ao paciente em parada cardiorrespiratória, um sobre aleitamento materno e seis sobre suporte básico de vida. Um dos participantes referiu não se lembrar da temática.

Sobre o game, o cenário é apresentado ao jogador, que escolhe entre duas situações de emergência para dar início ao jogo. Essas duas situações são histórias narradas pelo game e que o jogador deve utilizar objetos no cenário, ações e interações com outros personagens para avançar nas etapas, além de responder corretamente às questões que surgem.

Os resultados são apresentados com base na validação do game pelos profissionais, por meio da aplicação do instrumento EGameFlow. Além disso, a partir da análise dos dados oriundos da atividade de grupo focal, estes foram consolidados em duas categorias temáticas: 1) Dificuldades vivenciadas pela equipe no uso do Reanimabebê; 2) Reanimabebê: inovação tecnológica e realismo como estratégias para a educação permanente.

\section{Avaliação da equipe de saúde por meio do instrumento Egameflow}

Por meio da utilização do instrumento EGameFlow, implementou-se a validação do game pelos profissionais. As notas médias das dimensões estão apresentadas na tabela 1.

Tabela 1 - Média das avaliações do instrumento EGameFlow do Reanimabebê. Maringá, PR, Brasil, 2019

\begin{tabular}{lc}
\hline Avaliações & Média \\
\hline Concentração & 6,4 \\
C1 - O jogo prende minha atenção? & 7,0 \\
C2 - Apresenta conteúdo que estimula minha atenção? & 7,0
\end{tabular}


C4 - Nenhuma distração da tarefa é destacada?

C5 - No geral, consigo ficar concentrado no jogo?

C6 - Não sou distraído de tarefas nas quais deveria me concentrar?

C7 - Não sou sobrecarregado com tarefas que parecem sem importância?

C8 - A carga de trabalho do jogo é adequada?

Média Final

Desafios

H1 - Aproveito o jogo sem ficar entediado ou ansioso?

H2 - Dificuldade é adequada?

H3 - Existem “dicas” que ajudam na tarefa?

H4 - Apresenta suporte on-line que ajuda na tarefa?

H5 - Apresenta vídeo ou áudio que ajudam na tarefa?

H6 - Minhas habilidades aumentam conforme o jogo avança?

H7 - Sou motivado pela melhora das minhas habilidades?

H8 - Os desafios aumentam conforme minhas habilidades aumentam?

H9 - Apresenta novos desafios em um ritmo adequado?

H10 - Apresenta diferentes níveis de desafios que se adaptam aos diferentes jogadores?

Média Final

Autonomia

A1 - Tenho sensação de controle do menu?

A2 - Tenho sensação de controle sobre funções e objetos?

A3 - Tenho sensação de controle sobre as interações entre funções e objetos?

A4 - É possível cometer erros que impedem o avanço do jogo?

A5 - Posso me recuperar de qualquer erro cometido?

A6 - Sinto que posso usar quaisquer estratégias?

A7 - Tenho sensação de controle e impacto sobre o jogo?

A8 - Sei o próximo passo no jogo?

A9 - Tenho sensação de controle sobre o jogo?

Média Final

Clareza dos objetivos

G1 - Objetivos gerais apresentados no início do jogo?

G2 - Objetivos gerais apresentados claramente?

G3 - Objetivos intermediários apresentados no local apropriado? 
G4 - Objetivos intermediários apresentados claramente?

G5 - Eu entendo os objetivos do aprendizado através do jogo?

Média Final

Feedback

F1 - Recebo feedback do meu progresso no jogo?

F2 - Recebo feedback imediato das minhas ações?

F3 - Sou notificado sobre novas tarefas imediatamente?

F4 - Sou notificado sobre novos eventos imediatamente?

F5 - Recebo informação sobre sucesso ou falha de objetivos intermediários

imediatamente?

F6 - Recebo informação sobre o meu status, como nível ou pontuação?

Média Final

Imersão

I1 - Esqueço do tempo enquanto jogo?

I2 - Esqueço das coisas ao meu redor enquanto jogo?

I3 - Esqueço dos problemas do dia a dia enquanto jogo?

I4 - Sinto uma noção de tempo alterada?

I5 - Posso ficar envolvido com o jogo?

I6 - Me sinto emocionalmente envolvido com o jogo?

I7 - Me sinto visceralmente envolvido com o jogo?

Média Final

MElhoria do conhecimento

$\mathrm{K} 1$ - O jogo melhora meu conhecimento?

K2 - Capto as ideias básicas do conteúdo apresentado? $\quad 6,8$

K3 - Tento aplicar o conhecimento no jogo? $\quad 6,8$

K4 - O jogo motiva o jogador a integrar o conteúdo apresentado? 7,0

K5 - Quero saber mais sobre o conteúdo apresentado? $\quad 7,0$

Média Final

Fonte: Elaborado pelos autores, 2019.

$$
\text { Com relação ao domínio }
$$

"Concentração", a equipe pontuou nota média de 6,5, indicando a oferta de um enredo com uma sequência coerente e agradável/motivadora.

O domínio "Desafio" recebeu nota média final de 6,3, denotando a existência de 
situação desafiadora, capaz de motivar o jogador a permanecer no jogo. O item que recebeu menor nota foi com relação ao suporte on-line, visto que o jogo não proporcionava tal subsídio.

Com relação à "Autonomia", os sujeitos consideraram possuir autonomia sobre o game, com nota final 6,1. Ao serem questionados enquanto preenchiam essa parte do instrumento, referiram que existiram dúvidas sobre o que era para ser feito em determinadas cenas. Algumas dúvidas eram sobre o acesso ao "menu" ou recuperação de erros cometidos durante o jogo. Por se tratar de uma versão Alpha, os erros e/ou sugestões podem, a posteriori, serem realinhados no jogo.

A nota atribuída ao domínio "Clareza dos Objetivos" foi 6,6. Embora não se tenha previsto a existência de uma escala de desempenho/pontuação de cada jogador, a categoria "Feedback" recebeu nota 6,6 , indicando que o game apresentou retornos imediatos (feedbacks positivos e negativos) após as ações do jogador por meio de avisos automáticos descrevendo se a conduta tomada foi correta ou não.

Os sujeitos atribuíram nota 6,3 em relação ao domínio "Imersão", sugerindo que o jogo promove o envolvimento do indivíduo, levando-o por vezes a desligar-se do ambiente ao redor enquanto joga, garantindo sua plena imersão e concentração. A categoria "Interação social" não pôde ser avaliada, já que o jogo não fornece subsídios para este item.

Finalmente, no que tange ao domínio "Melhoria do Conhecimento", a nota média final foi de 6,9, indicando a percepção de aquisição de conhecimentos pelos participantes. De modo geral, verificou-se uma avaliação positiva do game, pautada especialmente em seu caráter inovador, lúdico, e adequado em termos de sua pertinência técnico-científica e em relação à sua fidelidade ao contexto/cenário assistencial retratado.

\section{Dificuldades vivenciadas pela equipe no uso do Reanimabebê}

Em complementariedade à validação pelo instrumento EGameFlow, os sujeitos mencionaram duas principais dificuldades durante a utilização do jogo Reanimabebê. Conforme relatado por quatro pessoas, a dinâmica do jogo não deixava claro de que forma se daria a ventilação do bebê. Isto porque se tratava de um estágio do game no qual o jogador permanecia paralisado, o que, na opinião dos participantes, poderia induzir ao abandono do jogo.

Até então, a cena era acompanhada por um cronômetro ou temporizador e o jogador era solicitado a ventilar o bebê com bolsa válvula-máscara. Em função desta avaliação negativa dos profissionais, a pesquisadora, em parceria com a equipe de informática responsável, optou por reformular 
esta etapa do jogo, com a incorporação de uma instrução mais clara acerca do que o jogador deveria fazer:

Eu fiquei travada na parte da ventilação, eu não acertava o tempo certo entre uma e outra 'ventilada' com ambu (bolsa válvula-máscara) (e8).

No que tange à medicação durante a parada três sujeitos relataram dificuldade no preparo e administração de adrenalina endovenosa. Tal achado foi evidenciado nos discursos dos sujeitos de nível técnico:

Eu apanhei bastante foi com a medicação. Querendo ou não, quem faz essa parte é o enfermeiro. Achei bem bacana porque ativa a curiosidade da pessoa (e6).

Embora o assunto tenha gerado discussões, os próprios profissionais reconheceram a necessidade do conhecimento compartilhado, como condição sine qua non à otimização do trabalho colaborativo e interprofissional na situação de atendimento ao bebê em parada cardiorrespiratória:

É importante conhecermos também sobre a medicação (e1). Precisamos saber tudo o que está acontecendo, toda a equipe trabalhando junto para o melhor atendimento ao bebê (e3).

Em meio às reflexões no grupo focal, algumas sugestões emanaram do grupo, tais como a elaboração de mais casos/fases; inserção de pontuação ou escala de desempenho no jogo e a possibilidade de visualização do escore dos demais jogadores.
A questão da pontuação/competitividade foi apontada com base na justificativa de que esta promoveria um incentivo/estímulo entre os jogadores:

Mesmo que cada um esteja na sua casa... poderia mostrar quem está jogando em tempo real ou mesmo uma escala de pontuação, sabe? (e2). Ver a pontuação dos colegas gera aquela vontade na gente: Não, meu bebê vai viver, espera aí, vou fazer tudo certo (e9).

Outros desfechos foram propostos com o objetivo de tornar o game ainda mais palpável, como o desenvolvimento de novas situações para serem solucionadas pelo jogador:

Eu acho que deveria ter mais fases. Embora já tenha um desfecho, poderia ter mais de um, por exemplo, a criança evoluir para óbito quando não acertamos. Cada um teria uma experiência diferente (e8). Eu senti assim: Nossa, eu queria muito que continuasse. Porque tem uma série de possibilidades pra acontecer (e10).

Um ponto bastante discutido foi em relação a ideias de novos games para o público em geral. Alguns participantes sugeriram o desenvolvimento de um serious game com a temática "Primeiros Socorros" para ser disponibilizado à população:

Até para as nossas crianças seria muito bom! Talvez, se fosse algo sobre primeiros socorros, coisas básicas, porque eles se interessam pelo jogo, chama a atenção (e6). $\mathrm{Eu}$ acho que você deveria desenvolver mais casos nesse jogo e também outros temas em outros 
aplicativos, não só para profissional de saúde, mas também para pessoas leigas. É muito didático! (e10).

\section{Reanimabebê: inovação tecnológica e realismo como estratégias para a educação permanente}

A construção de cenários de jogos com base nas realidades e contextos assistenciais a que se direcionam propicia níveis de realismo que facilitam a imersão dos sujeitos no game, aumentando o envolvimento e otimizando os resultados da ação educativa:

Desenhamos o cenário da emergência naquela outra reunião. Eu achei que as imagens são muito parecidas com a nossa realidade. Até o local onde fica o monitor foi retratado (e1).

A situação de chegada da criança grave à emergência foi avaliada como muito similar à realidade vivenciada. Ademais, os sujeitos referiram que existe sequência lógica das etapas do atendimento ao bebê em parada cardiorrespiratória, tornando o game bastante adequado e didático:

O Reanimabebê tem uma lógica sequencial de acontecimentos (e8). Por exemplo, na intubação, não aparece a criança já intubada, mostra o tubo, mostra colocando os eletrodos, ficou muito bom! (e4).

Além de seu caráter educativo, o jogo foi definido ainda como estratégia inovadora, prática, original, e agregador de um componente de entretenimento ao processo ensino-aprendizagem:

A ideia é sensacional. Prático. Muito bom (e4). Essa forma de aprender é muito didática e inovadora (e2). Apesar do caráter educacional, me instigou como uma brincadeira (e7).

\section{DISCUSSÃO}

Os resultados do estudo demonstram que o uso de abordagens tecnológicas dentro dos serviços de saúde podem trazer resultados positivos para a prática, de modo a capacitar de forma dinâmica os profissionais de saúde. Estudo realizado na França, em que alunos foram treinados com um serious game voltado às emergências obstétricas, demonstrou que foi possível o aprendizado ativo, já que o sujeito era imerso em um ambiente que recriava o cenário obstétrico próximo à realidade, proporcionando enfrentar situações de emergência que demandavam tomada de decisão rápida e baseada em conhecimentos efetivos ${ }^{(17)}$.

$$
\text { Pesquisa desenvolvida na }
$$

Universidade Federal de Pernambuco, onde se criou um serious game direcionado aos adolescentes com objetivo de promover reflexões sobre saúde sexual e reprodutiva. Após a validação do jogo com o público-alvo e também com profissionais da respectiva área, concluiu-se que foi possível a imersão no jogo porque houve uma aproximação do game com o dia a dia dos adolescentes, através do uso de linguagem específica 
utilizada pelos personagens, trazendo o jogo próximo da realidade ${ }^{(18)}$.

Neste estudo, buscou-se incluir os sujeitos no desenvolvimento do game com o intuito de tornar a aprendizagem próxima da realidade local e, dessa forma, bastante fiel ao contexto de atuação do público-alvo. Além de se tratar de uma ferramenta inovadora, didática e fácil de manusear, o game apresenta facilidades na tomada de decisão, por se tratar de uma simulação bastante realista, porém em ambiente livre de estresse. Esta conclusão ratifica diversos estudos na esfera nacional e internacional $^{(17,19-20)}$.

Alguns sujeitos referiram problemas na etapa na qual era necessário ter conhecimento do preparo e administração da adrenalina. Inicialmente, isto foi justificado pelo fato de tal atribuição ser exclusiva ao enfermeiro, porém, após discussão em grupo, houve reconhecimento acerca da importância de todos da equipe partilharem conhecimentos sobre o manejo completo do bebê em parada cardiorrespiratória, independente da categoria profissional.

Vale ressaltar que a equipe de enfermagem é uma das primeiras a identificar que algo não está bem com o bebê, portanto manter o ambiente e os materiais adequados, bem como saber manejá-los de forma correta e administrar os medicamentos necessários, é fundamental para que as decisões tomadas durante $o$ atendimento de emergência sejam bem realizadas e contemplem a participação de todos, de modo que o objetivo seja sempre reestabelecer a saúde do paciente em atendimento, em especial no que tange à população pediátrica ${ }^{(21)}$.

Outro ponto importante destacado pelo grupo referiu-se à ventilação no bebê. Segundo os sujeitos, esse momento não estava claro, pois faltavam detalhes de comando do game sobre o que fazer na ocasião para dar continuidade ao jogo. Optou-se por aperfeiçoar esta etapa, pois, a equipe que irá utilizar o produto deve testá-lo e expor sugestões e implicações relacionadas à sua realidade para que o escopo primordial (proporcionar a aprendizagem) seja consolidado $^{(8)}$.

Com relação à avaliação dos sujeitos, através do instrumento EGameFlow, pode-se inferir que todos os domínios (concentração, desafio, autonomia, clareza dos objetivos, feedback, imersão e melhoria do conhecimento) apresentaram pontuação apropriada, denotando o potencial de utilização do game. As limitações do estudo dizem respeito a não realização de avaliações comparativas de aprendizagem dos profissionais, antes e após a utilização do game, além disso o número restrito de profissionais que participaram do estudo devido baixa quantidade de recursos humanos naquele local e recusas. Sugere-se, portanto, o desenvolvimento de novos estudos que procedam à aplicação e avaliação desta ferramenta em outras realidades. 


\section{CONSIDERAÇÕES FINAIS}

Conclui-se que o serious game Reanimabebê apresentou boa aceitação do público-alvo, visto que os sujeitos relataram enfaticamente este achado, mostrando-se aplicável em unidade de pronto atendimento.

Destaca-se ainda se tratar de uma ferramenta inovadora, didática e que guarda bastante proximidade em relação à realidade vivenciada. Os sujeitos relataram que a ferramenta é eficaz para a aprendizagem e recomendam seu uso para outras populações e contextos assistenciais.

\section{REFERÊNCIAS}

1. Johnsen HM, Fossum M, VivekanandaSchmidt P, Dphil, CPsychol, Fruhling A et al. Developing a serious game for nurse education. J Gerontol Nurs [Internet]. 2018 [Cited 2021 jan 7]; 44(1). Available from: https://pubmed.ncbi.nlm.nih.gov/29355884/. doi: $\quad$ https://doi.org/10.3928/0098913420171213-05

2. Fonseca LMM, Aredes NDA, Fernandes AM, Batalha LMC, Apóstolo JMA, Martins JCA et al. Computer and laboratory simulation in the teaching of neonatal nursing: innovation and impact on learning. Rev Latino-Am Enfermagem [Internet]. 2016 [Cited 2021 jan 7]; 24:e2808. Available from: https://www.scielo.br/pdf/rlae/v24/01041169-rlae-24-02808.pdf. doi: https://dx.doi. org/10.1590/1518-8345.1005.2808

3. Mather C, Cummings E. Issues for deployment of mobile learning by nurses in Australian Healthcare Settings. Stud Health Technol Inform [Internet]. 2016 [Cited 2021 jan 8]; 225:277-81. Available from: https://pubmed.ncbi.nlm.nih.gov/27332206/. doi: 10.3233/978-1-61499-658-3-277
4. Dias JD, Tibes CMS, Fonseca LMM, ZemMascarenhas SH. Use of serious games for coping with childhood obesity: Integrative literature review. Texto Contexto Enferm [Internet]. 2017 [Cited 2021 jan 7]; 26(1):e3010015. Available from: https://www.scielo.br/pdf/tce/v26n1/1980-

265X-tce-26-01-e3010015.pdf. http://dx.doi.org/10.1590/010407072017003010015

5. Creutzfeldt J, Hedman L, Fellander-Tsai L. Cardiopulmonary resuscitation training by avatars: a qualitative study of medical students experiences using a multiplayer virtual world. JMIR Serious Game [Internet]. 2016 [Cited 2021 jan 6]; 4(2): e22. Available from: https://games.jmir.org/2016/2/e22/. doi: http://dx.doi.org/10.2196/games.6448

6. Deguirmendjian SC, Miranda FM, Mascarenhas SHZ. Serious game developed in health: integrative literature review. J Health Inform [Internet]. 2016 [Cited 2020 oct 19]; 8(3):110-6. Available from: https://pesquisa.bvsalud.org/portal/resource/pt /biblio-831881

7. Panaggio BZ, Baranauskas MCC. From consumers to co-authors: exploring the participatory design of tangible technology in an educational context. Braz J Comput Educ [Internet]. 2019 [Cited 2020 oct 20]; 27(2):91-111. Available from: https://www.brie.org/pub/index.php/rbie/article/view/v27n02 91111. doi:

http://dx.doi.org/10.5753/RBIE.2019.27.02.91

8.Carneiro KKC, Moraes-Filho IM, Santos OP, Arantes AA, Félis KC, Guilherme IS, et al. Simulação realística como instrumento no processo de ensino-aprendizagem de enfermagem. REVISA [Internet]. 2019 [Cited 2020 nov 2]; 8(3):273-84. Available from: http://revistafacesa.senaaires.com.br/index.ph $\mathrm{p} /$ revisa/article/view/423/314. doi: https://doi.org/10.36239/revisa.v8.n3.p273a28 4

9. Bonfim CJL, Mombach JG, Martins ABN, 
Sousa JKLL. Design Participativo: Uma Experiência de Criação de Aplicativos com Meninas. RSC [Internet]. 2018 [Cited 2021 abr 06]; 8(2):402-417. Available from: https://revistas.unifacs.br/index.php/rsc/article /view/5780/3669.

10. American Heart Association (AHA). Pediatric Advanced Life Support Provider Manual (PALS). 4. ed. USA: American Heart Association; 2016.

11. Polit DF. Fundamentos de pesquisa em enfermagem: Avaliação de evidências para a prática da enfermagem. 9. ed. Porto Alegre: Artmed; 2018.

12. Hui CS, Yih WW, Dennison J. Validation of EGameFlow: A self-report scale for measuring user experience in video game play. Comput Entertain [Internet]. 2018 [Cited 2021 jan 20]; 16(3). Available from: https://dl.acm.org/doi/10.1145/3238249. doi: https://doi.org/10.1145/3238249

13. Lima T, Barradas AF, Barros AK, Viana D, Bottentuit JBJ, Rivero L. Avaliando um Jogo Educacional para o Ensino de Inteligência Artificial - Qual Metodologia para Avaliação Escolher? In: Workshop sobre educação em computação 2020; Porto Alegre, RS: Sociedade Brasileira de Computação. [Internet]. 2020 [Cited 2021 abr 06]. P. 1-5. Available from: https://sol.sbc.org.br/index.php/wei/article/vie w/11131/11002. doi: https://doi.org/10.5753/wei.2020.11131.

14 Dias JD, Mekaro MS, Lu JKC, Otsuka JL, Fonseca LMM et al. Serious game development as a strategy for health promotion and tackling childhood obesity. Rev Latino-Am Enfermagem [Internet]. 2016 [Cited 2021 jan 7]; 24: p.e2759. Available from:

https://www.scielo.br/pdf/rlae/v24/01041169-rlae-24-02759.pdf. doi:10.1590/15188345.1015.2759.

15. Silveira MS, Cogo ALP. The contributions of digital technologies in the teaching of nursing skills: an integrative review. Rev Gaúcha Enferm [Internet]. 2017 [Cited 2021 jan 6]; 38(2):e66204. Avalable from:

https://www.scielo.br/pdf/rgenf/v38n2/en_01 02-6933-rgenf-1983-144720170266204.pdf. doi: $\quad$ http://dx.doi.org/10.1590/19831447.2017.02.66204

16. Bardin L. Análise de conteúdo. 1. ed. São Paulo: Edições 70; 2016.

17. Gautier EJD, Bot-Robin V, Libessart A, Doucede G, Cosson M, Rubod C. Design of a serious game for handling obstetrical emergencies. JMIR Serious Game [Internet]. 2016 [Cited 2021 jan 6]; 4(2):e21. Available from: https://games.jmir.org/2016/2/e21/. doi: http://dx.doi.org/10.2196/games.5526

18. Monteiro RJS, Oliveira MPCA, Belian RB, Lima LS, Santiago ME, Gontijo DT. DECIDIX: meeting of the Paulo Freire pedagogy with the serious games in the field of health education with adolescentes. Ciênc Saúde Colet [Internet]. 2018 [Cited 2021 jan 7]; 23(9):2951-62. Available from: https://www.scielo.br/pdf/csc/v23n9/en_1413 -8123-csc-23-09-2951.pdf. doi: http://dx.doi.org/10.1590/1413-

81232018239.12782018

19. Benitez JLF, Pascual JLG, Sanz EP. DietaDialisisQuiz. A multiplayer competitive serious game for learning during dialysis. Enferm Nefrol [Internet]. 2017 [Cited 2020 dec 19]; 20(4):378-81. Available from: http://dx.doi.org/10.4321/s2254-

28842017000400012.

doi: http://dx.doi.org/10.4321/s225428842017000400012

20. Leal F, Martins PC, Torres AF, Queiroz JA, Montevechi JAB. Learning lean with lego: deeloping and evaluating the efficacy of a serious game. Prod [Internet]. 2017 [Cited 2020 dec 19]; 27:e20162227. Available from: https://www.scielo.br/pdf/prod/v27nspe/01036513-prod-0103-6513222716.pdf. doi: http://dx.doi.org/10.1590/0103-6513.222716 
21. Melo K, Silva T, Andrade J, Ribeiro J, Bandeira L, Silva M. Reanimação neonatal. REAID [Internet]. 2021 [Cited 2021 set 08]; 95(34):e-21066. Available from: https://revistaenfermagematual.com/index.ph $\mathrm{p} /$ revista/article/view/974. doi: https://doi.org/10.31011/reaid-2021-v.95n.34-art.974

\section{Autor correspondente}

Roberta Tognollo Borotta Uema. Rua Antonio Valdir Zanuto, número 53. Jardim Novo Horizonte, Maringá, PR. CEP: 87010100. E-mail: robertaborotta@ hotmail.com

Submissão: 2021-08-04

Aprovado: 2021-10-11 